\title{
Homeobox B3 promotes tumor cell proliferation and invasion in glioblastoma
}

\author{
$\mathrm{KE} \mathrm{XU}^{1 *}, \mathrm{CHUN}^{2} \mathrm{QIU}^{2 *}$, HUA PEI $^{1}$, MUHAMMAD AAMER MEHMOOD $^{3,4}$, \\ HUAMIN WANG ${ }^{1}$, LIANG LI $^{1}$ and QIANFENG XIA ${ }^{5}$
}

\begin{abstract}
${ }^{1}$ Department of Immunology, School of Tropical and Laboratory Medicine, Hainan Medical University; ${ }^{2}$ Department of Oncology, Hainan Provincial People's Hospital, Haikou, Hainan 571101, P.R. China; ${ }^{3}$ Department of Bioinformatics and Biotechnology, Government College University Faisalabad, Faisalabad, Punjab 38000, Pakistan; ${ }^{4}$ School of Life Sciences and

Biotechnology, Shanghai Jiao Tong University, Shanghai 200240; ${ }^{5}$ Key Laboratory of Tropical Biomedicine, and

Faculty of Tropical Medicine and Laboratory Medicine, Hainan Medical University, Haikou, Hainan 571101, P.R. China
\end{abstract}

Received October 27, 2016; Accepted November 10, 2017

DOI: $10.3892 / \mathrm{ol} .2018 .7750$

\begin{abstract}
Glioblastoma (GBM) is the most aggressive brain tumor in adults with the highest mortality rate. Despite advances achieved in treatment and research, the median survival for patients with GBM remains $<1.5$ years. This figure prompted the present study to identify novel genes associated with GBM development and progression to ultimately improve GBM treatment. The current study sought to determine the role of homeobox B3 (HOXB3) in GBM cell invasion and proliferation. HOXB3 was highly expressed in GBM tissues and glioma cell lines. To establish in vitro cell models for investigation, U87-MG and U251-MG, two typical GBM cells, were selected to generate corresponding cells lines that constitutively silenced HOXB3 expression using a lentivirus-mediated RNA interference approach. The results of the knockdown revealed that glioma cells stably expressing HOXB3 short hairpin RNA exhibited significantly decreased proliferation levels when compared with untransfected cells. The effect of HOXB3 on glioma cell invasion was also examined. Silencing of HOXB3 resulted in a marked reduction in invasiveness. Furthermore, HOXB3 silencing led to the upregulation of E-cadherin and downregulation of mesenchymal markers, $\mathrm{N}$-cadherin and vimentin. Taken together, the findings of the present study indicate that HOXB3 promotes cell proliferation and invasion.
\end{abstract}

Correspondence to: Dr Qianfeng Xia, Key Laboratory of Tropical Biomedicine, and Faculty of Tropical Medicine and Laboratory Medicine, Hainan Medical University, 3 Xueyue Road, Haikou, Hainan 571101, P.R. China

E-mail: xiaqianfeng@sina.com

*Contributed equally

Key words: homeobox B3, U251-MG cells, U87-MG cells

\section{Introduction}

Glioblastoma multiforme (GBM) is the most common type of primary malignant brain tumor in adults worldwide (1). Despite advance in its treatment, prognosis of patients with GBM remains poor: Patients have a median survival time of 1 year and $<5 \%$ of patients survive for 5 years (2). As tumor cells can infiltrate into adjacent normal brain tissues, allowing them to evade therapeutic interventions and proliferate rapidly; tumor cell proliferation and invasion are regarded as primary causes of poor prognosis in patients with GBM. Therefore, it is essential to identify genes that contribute to GBM cell proliferation and invasion.

Homeobox genes are regulatory genes that encode transcription factors during embryogenesis and normal development, in which they regulate cell differentiation, and proliferation (3). There are $>20$ subclasses of homeobox genes; most notable among these is homeobox (HOX) gene family that consists of 39 genes (4). These genes are subdivided into four groups: A, B, $\mathrm{C}$ and D $(5,6)$. HOX genes are reportedly implicated in tumor progression. HOXA7, HOXB7 and HOXA10 were highly expressed in human lung cancer (7). HOXD9 mRNA was present in cervical cancer, but absent in the normal cervix $(8,9)$. Overexpression of HOXB6, HOXB8, HOXC8, and HOXC9 were observed in human colon cancer (10). High expression of Hoxa1, along with the downregulation of Hoxc9, was observed in the neoplastic mouse mammary gland (11). Upregulation of HOXD3 and HOXD4, and the concomitant downregulation of HOXA11 and HOXB5, were observed in human breast cancer (9). In addition to the from the aforementioned HOX members, HOXB3 has recently attracted attention as its altered expression has been observed in a variety of cancer types. For instance, HOXB3 was upregulated in primary prostate cancer tissues compared with normal prostate tissues. This upregulation was associated with higher Gleason grades and with poor patient survival outcome (12). Overexpression of HOXB3 in prostate cancer $\mathrm{LNCaP}$ cells promoted cellular proliferation and migration (12). Immunocytochemical analysis of lung carcinoma tissues exhibited high expression of HOXB3 (13). Silencing of HOXB3 in non-small cell lung cancer A549 
cells resulted in decreased growth, whereas overexpression of HOXB3 in non-small cell lung cancer NCI-H1437 cells markedly increased tumor growth rate (14). Although HOXB3 has been investigated in multiple cancer types, its role in GBM remains unknown. The present study was therefore designed to investigate whether HOXB3 contributes to cell proliferation and invasion in GBM.

\section{Materials and methods}

Cell lines and cell culture. The glioma cell line U251-MG was obtained from the Cell Bank of the Chinese Academy of Sciences (Shanghai, China), and U87-MG from American Type Culture Collection (Manassas, VA, USA). The two human glioma cell lines were cultured in Dulbecco's modified Eagle's medium Dulbecco's modified Eagle's medium (DMEM; Gibco; Thermo Fisher Scientific, Inc., Waltham, MA, USA) containing $2 \mathrm{mM}$ glutamine, $10 \%$ fetal calf serum (FBS) (both Thermo Fisher Scientific, Inc.), $100 \mathrm{U} / \mathrm{ml}$ penicillin and $100 \mu \mathrm{g} / \mathrm{ml}$ streptomycin (Sigma-Aldrich; Merck KGaA, Darmstadt, Germany). Cells were maintained in an incubator at $37^{\circ} \mathrm{C}$ in a $5 \% \mathrm{CO} 2$ atmosphere. HEB cells were cultured at $37^{\circ} \mathrm{C}$ for 3 days in DMEM (Gibco; Thermo Fisher Scientific, Inc.) containing $2 \mathrm{mM}$ glutamine, $10 \% \mathrm{FBS}$ (both Thermo Fisher Scientific, Inc.), $100 \mathrm{U} / \mathrm{ml}$ penicillin (Sigma-Aldrich; Merck KGaA) and $100 \mu \mathrm{g} / \mathrm{ml}$ streptomycin (Sigma-Aldrich; Merck KGaA).

Tumor samples. Glioblastoma specimens were obtained from patients (4 male and 2 female, ranging from 44-67 years old and admitted between September 2015 and September 2016) who underwent surgical resection at Hainan Provincial People's Hospital. Normal brain specimens were acquired from 3 patients undergoing surgery for epilepsy and were reviewed to verify the absence of tumor. All samples were collected under protocols approved by the Institutional Review Board of Hainan Provincial People's Hospital and each patient provided written informed consent for inclusion in the present study.

Establishment of stably transfected HOXB3-knockdown cell lines. DNA oligos designed by the BLOCK-iT RNAi designer (Invitrogen; Thermo Fisher Scientific, Inc.) encoding human HOXB3-short hairpin RNAs (sequence, 5'-CGGTAAAGC CCACCAGAAT-3') (HOXB3-knockdown) were synthesized and cloned into the pHY-LV-KD1.1 vector (Shanghai Hanyu Biotechnology Co., Ltd., Shanghai, China) to generate pHY-FoxO3a-KD. A vector expressing short hairpin RNA (shRNA) targeted against an irrelevant sequence (shRNA-NC) was used as a negative control. The Trans-Lentiviral Packaging system and ViraPower Lentiviral Expression system (Invitrogen; Thermo Fisher Scientific, Inc.) were used to produce shRNA. These constructs were co-transfected with 15 ug packaging plasmids (Invitrogen; Thermo Fisher Scientific, Inc.) into 293 cells using Lipofectamine 2000 (Invitrogen; Thermo Fisher Scientific, Inc.), according to the manufacturer's protocol, and viral particles were harvested $48 \mathrm{~h}$ later. Lentiviruses containing shRNAs were used to silence FoxO3a expression in U87-MG and U251-MG cells. Stably transfected cells that consecutively silence HOXB3 were generated using one week of puromycin selection.
Cell proliferation. Cell counts were determined using Cell Counting Kit-8 (Dojindo Molecular Technologies, Inc., Kumamoto, Japan). A total of $1 \times 10^{3} \mathrm{GBM}$ cells were plated onto 96-well culture plates in triplicate, and cell growth was determined daily for 5 days using a tetrazolium salt-based colorimetric assay (Dojindo Molecular Technologies, Inc.) according to the manufacturer's protocol. Absorbance was measured at $450 \mathrm{~nm}$. Three independent experiments were performed.

Transwell invasion assay. For the Transwell assay, $2 \times 10^{4}$ stably transfected cells were plated into 24-well Boyden chambers (Corning Incorporated, Corning, NY, USA) with an $8-\mu \mathrm{m}$ pore polycarbonate membrane coated with $30 \mu \mathrm{g}$ Matrigel (BD Biosciences, San Jose, CA, USA). Cells were plated in the upper chamber with $200 \mu \mathrm{l}$ of serum-free medium (DMEM; Gibco; Thermo Fisher Scientific, Inc.), and the medium containing $20 \%$ FBS was added to the lower chamber to serve as a chemoattractant. After $36 \mathrm{~h}$, the cells were washed three times with PBS. Non-invasive cells were removed from the upper well using cotton swabs, and the invasive cells were then fixed with paraformaldehyde for $15 \mathrm{~min}$ at room temperature, air-dried, and stained with $0.1 \%$ crystal violet for $15 \mathrm{~min}$ at room temperature. The results were observed under a light microscope in 5 predetermined fields (magnification, x200).

Western blot analysis. Cells were lysed in radioimmunoprecipitation assay buffer (50 mM Tris- $\mathrm{HCl} \mathrm{pH}$ 8.0,1 mM EDTA $\mathrm{pH} 8.0,5 \mathrm{mM}$ DTT, $2 \% \mathrm{SDS}$ ), and protein concentration was determined using a BCA assay (Beyotime Institute of Biotechnology, Haimen, China). Total protein (30 $\mu$ g per lane) was resolved using a 10\% SDS-PAGE and electrotransferred to polyvinylidene fluoride membranes (Invitrogen; Thermo Fisher Scientific, Inc.), then blocked with 5\% non-fat dry milk in Tris-buffered saline, $\mathrm{pH} 7.5$ for $1 \mathrm{~h}$ at room temperature. Membranes were incubated with primary antibodies overnight at $4^{\circ} \mathrm{C}$. The primary antibodies used were HOXB3 (dilution, 1:400; Santa Cruz Biotechnology, Inc., Dallas, TX, USA; cat. no. sc28606), N-cadherin (dilution, 1:800 Cell Signaling Technology, Inc., Danvers, MA, USA; cat. no. 4061), vimentin (dilution, 1:200; Cell Signaling Technology, Inc.: cat. no. 3932), E-cadherin (dilution, 1:1,000; Cell Signaling Technology, Inc.; cat. no. 3195) and $\beta$-actin (dilution, 1:4,000; ProteinTech Group, Inc., Chicago, IL, USA; cat. no. HRP60008). A horseradish peroxidase-conjugated $\mathrm{IgG}$ secondary antibody (dilution, 1:2,000; cat. no. ab6721, Abcam, Cambridge, MA, USA) was added and incubated at room temperature for $1 \mathrm{~h}$. Bound antibodies were detected using the BeyoECL system (cat. no. P0018; Beyotime Institute of Biotechnology), and densitometry was performed using ImageQuant 5.2 software (GE Healthcare Life Sciences, Little Chalfont, UK) was used for analysis.

Immunocytochemistry. Immunocytochemistry was performed to detect the expression of HOXB3. Initially, 5- $\mu \mathrm{m}$ sections of formalin-fixed paraffin-embedded tissues were dewaxed with xylene and rehydrated in 100 and $95 \%$ ethanol. Antigen retrieval was performed by boiling slides in $10 \mathrm{mM}$ sodium citrate buffer $\mathrm{pH} 6.0$ for $10 \mathrm{~min}$. Non-specific antibody binding in cells and slides was blocked by incubation with 
A

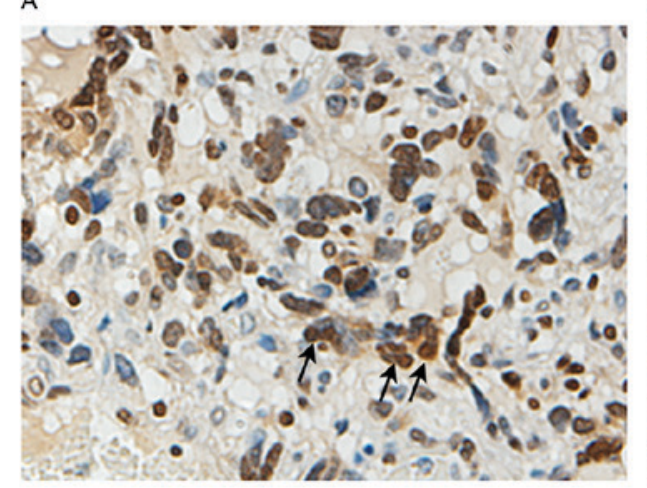

B

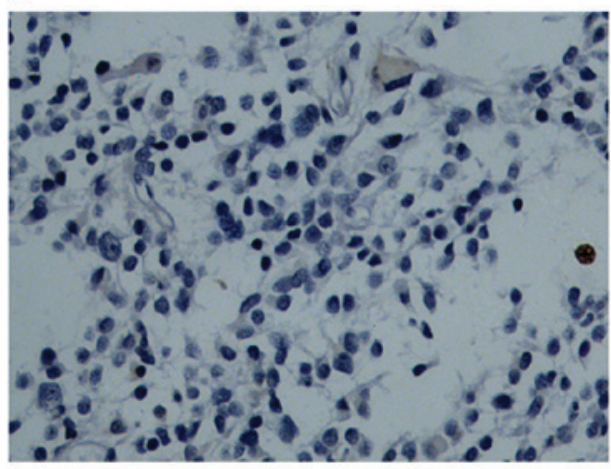

$\mathrm{C}$

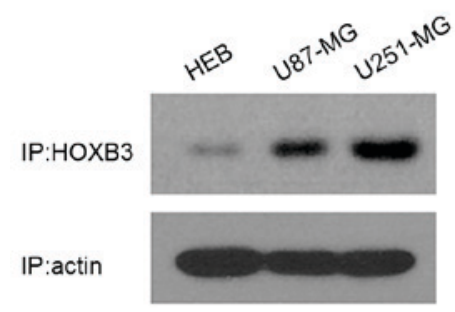

Figure 1. HOXB3 is highly expressed in primary GBM tumors and cell lines. (A) A representative immunohistochemical image of HOXB3-positive staining in GBM tissue. Human GBM patient and normal brain specimens were subjected to immunohistochemistry using a specific antibody against HOXB3. Strong positive staining for HOXB3 was detected in tumor specimens $(n=6)$. Magnification, $x 200$. (B) Negative staining was observed in normal human brain specimens (n=3). (C) High expression of HOXB3 in typical glioma cell lines. Western blot analysis of HOXB3 in glioma U87-MG and U251-MG cells and a normal glial cell HEB line revealed a significantly higher HOXB3 protein level in U87-MG and U251-MG cells than that of HOXB3 in HEB cells. As a normalizing control, $\beta$-actin was also detected in the same blot. GBM, glioblastoma; HOXB3, homeobox B3; HEB, human embryonal brain. Magnification, x200.

blocking buffer $(0.2 \%$ Triton X-100, $5 \%$ normal donkey serum, and $5 \%$ non-fat milk in Tris-buffered saline) for $1 \mathrm{~h}$ at room temperature prior to overnight incubation with affinity-purified specific primary antibody HOXB3 (dilution, 1:200; Santa Cruz Biotechnology, Inc.; cat. no. sc28606 ) in blocking buffer at $4^{\circ} \mathrm{C}$. Human GBM sections were then incubated with alkaline phosphatase-conjugated secondary antibody (dilution, 1:500; Cayman Chemical Company, Ann Arbor, MI, USA; cat. no. 13460-1) in the blocking buffer for $1 \mathrm{~h}$ at room temperature. Immunoreactivity was visualized by incubating the sections with 3,3-diaminobenzidine tetrahydrochloride (Dako; Agilent Technologies, Inc., Santa Clara, CA, USA) to produce brown precipitates. The slides were counterstained with hematoxylin at room temperature for $2 \mathrm{~min}$ for viewing negatively stained cells.

Statistical analysis. Data are presented in the graphs as the mean \pm standard deviation of three independent experiments. The differences among groups were determined using analysis of variance with post hoc contrasts determined using the Student-Newman-Keuls test, and comparisons between two groups were analyzed using the Student's t-test. $\mathrm{P}<0.05$ was considered to indicate a statistically significant difference. All statistical analyses were performed using GraphPad Prism 6 software (GraphPad Software, Inc., La Jolla, CA, USA).

\section{Results}

HOXB3 is highly expressed in U87-MG and U251-MG cells. To analyze the expression level of HOXB3 in GBM, 6 tissue samples of GBM (from surgical biopsy specimens) were initially examined using immunocytochemistry. Strongly positive staining was observed in human GBM patient specimens $(n=6)$; by contrast, HOXB3 was hardly detectable in normal brain samples $(n=3)$ (Fig. 1A and B). These results indicated that HOXB3 is predominantly present in cancerous tissue, but not in normal brain tissue. As U87-MG and U251-MG are typical in vitro models of GBM that can authentically recapitulate GBM cancerous phenotypes, specifically cell proliferation and invasion, these two cell lines were used to examine the expression level of HOXB3. As expected, high expression of HOXB3 was observed in U87-MG and U251-MG cells. By contrast, HEB cells exhibited mild expression of HOXB3 that was barely detectable by western blot analysis (Fig. 1C). These findings coincide with previous reports, which demonstrated that HOXB3 was highly expressed in cancerous cell lines $(13,15)$.

Silencing of HOXB3 inhibits cell proliferation in U87-MG and U251-MG cells. HOXB3 has been shown to serve a role in cell proliferation in multiple types of cancer, as demonstrated by its ability to induce cancer cell growth and tumor formation (16). To investigate the ability of HOXB3 to modulate cell proliferation in GBM, two typical GBM cell lines, U251-MG and U87-MG, were engineered using lentiviral gene transfer to express a specific shRNA against HOXB3. Upon transfection, GBM cells stably silencing HOXB3 were obtained through puromycin selection and assessed for their proliferation capacities. Fig. 2A depicts stably transfected U87-MG cells expressing significantly lower protein level of HOXB3 
A
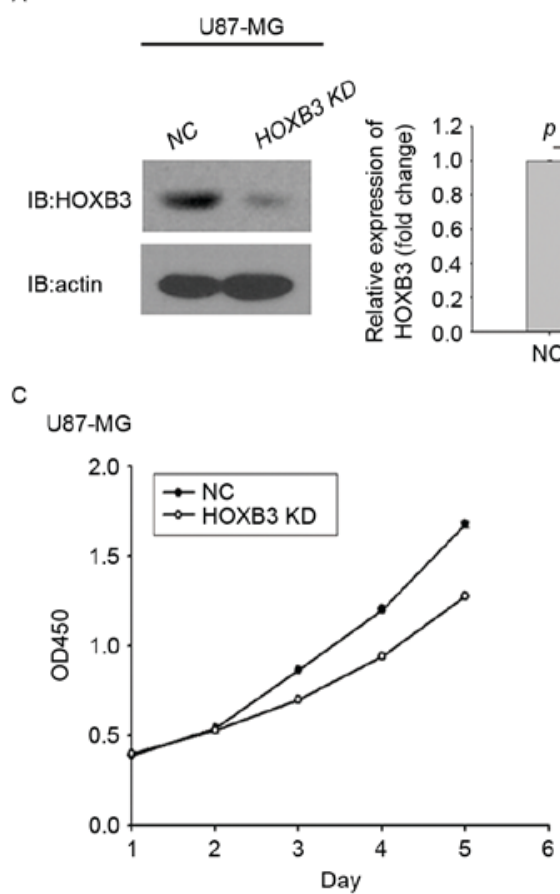

B

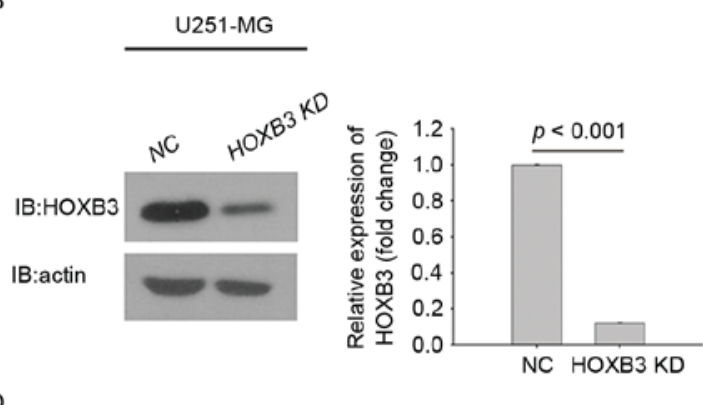

D

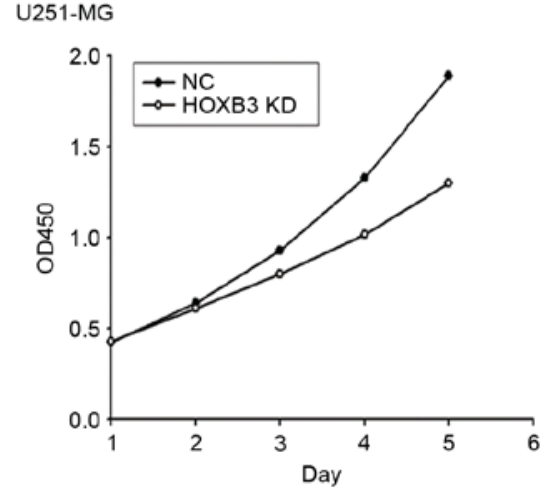

Figure 2. Silencing of HOXB3 inhibits GBM cell proliferation. (A) Western blot analysis measuring HOXB3 expression demonstrated that stably transfected U87-MG cells that constitutively silencing HOXB3 expression had been successfully generated. Densitometric analysis of the HOXB3 bands demonstrated a significantly lower expression in stably transfected U87-MG cells when compared to NC. (B) Western blot analysis of HOXB3 expression in HOXB3-KD U251-MG cells and its parental counterparts revealed that HOXB3-KD U251-MG cells had been successfully generated. Densitometric analysis of the HOXB3 bands demonstrated a significantly lower expression in HOXB3-KD U251-MG cells than in their parental counterparts. Cells were cultured in 96-well plates and analyzed using a Cell Counting kit-8 assay. Cell proliferation curves were plotted and used to depict cell growth in 5 days. (C) HOXB3-KD cells exhibited decreased proliferation relative to their parental counterparts. (D) HOXB3-KD U251-MG cells exhibited decreased proliferation relative to their parental counterparts. GBM, glioblastoma; HOXB3-KD, homeobox B3-knockdown; NC, negative control.

compared with the parental U87-MG cells, in which HOXB3 protein level were analyzed by western blot analysis. Similarly, this result was observed in stably transfected U251-MG cells (Fig. 2B).

These results indicated that the two stably transfected GBM cells had been successfully generated. Whether altered expression of HOXB3 had an effect on GBM cell proliferation was next investigated. Stably transfected U87-MG cells that consistently silenced endogenous HOXB3 exhibited lower cell proliferation capacity compared with parental U87-MG cells. This trend did not appear until day 3 , and this difference was further enhanced over time (Fig. 2C). Similarly, in the cohort of the U251 cells, the proliferative capacity of U251 cells with HOXB3-knockdown was severely diminished when compared with that determined for its parental cells. Notably, the turning point at which the growth rate of the parental cells exceeded that of the stably transfected cells engineered to silence HOXB3 also emerged on day 3 (Fig. 2D). It was evident that silencing of HOXB3 alone was able to confer to GBM cells, at least in the in vitro models in the present study, a reduced capacity for cell proliferation, indicating that HOXB3 serves an important role in GBM cell proliferation.

Silencing of HOXB3 inhibits cell invasion in U87-MG and $U 251-M G$ cells. In addition to promoting cell proliferation, HOXB3 has also been reported to be involved in cell invasion $(16,17)$. To determine the effect of HOXB3 on GBM cell invasion, a Matrigel invasion assay was performed to analyze the invasive capacities of the GBM cells stably expressing HOXB3 shRNA and their corresponding parental cells. Identical numbers of cells from the stably transfected and parental cohorts were plated into the upper chambers and incubated for $36 \mathrm{~h}$. Fig. 3A shows that HOXB3-silencing U87-MG cells exhibited a statistically significant reduction in cell invasion. Consistently, the stable silencing of HOXB3 in U251-MG cells also led to a significant reduction in cell invasion when compared with the parental U251-MG cells (Fig. 3B).

These findings indicate that HOXB3 alone is capable of modulating GBM cell invasion. Notably, silencing of HOXB3 in U87-MG and U251-MG cells changed some cells' elongated, fibroblastic morphology to become relatively rounded and cobbled (Fig. 3A and B), reminiscent of epithelial cells undergoing an epithelial-mesenchymal transition (EMT) $(18,19)$. Several prior studies have verified that upregulation of mesenchymal genes promotes cell invasion in GBM (20-24). It was therefore examined whether silencing of HOXB3 in GBM cells led to evident changes in the expression of EMT-associated genes. As expected, U87-MG cells that are stably silencing HOXB3 exhibited a marked decrease in the level of $\mathrm{N}$-cadherin and vimentin proteins, which are typical mesenchymal markers. By contrast, E-cadherin, which is a classical epithelial marker, was upregulated in the stably transfected U87-MG cells compared with its parental cells (Fig. 3C). A similar result was observed in the cohort of U251-MG cells (Fig. 3D). These findings demonstrate that HOXB3 is a critical driver of GBM invasion, and its effect on cell invasion may 
A
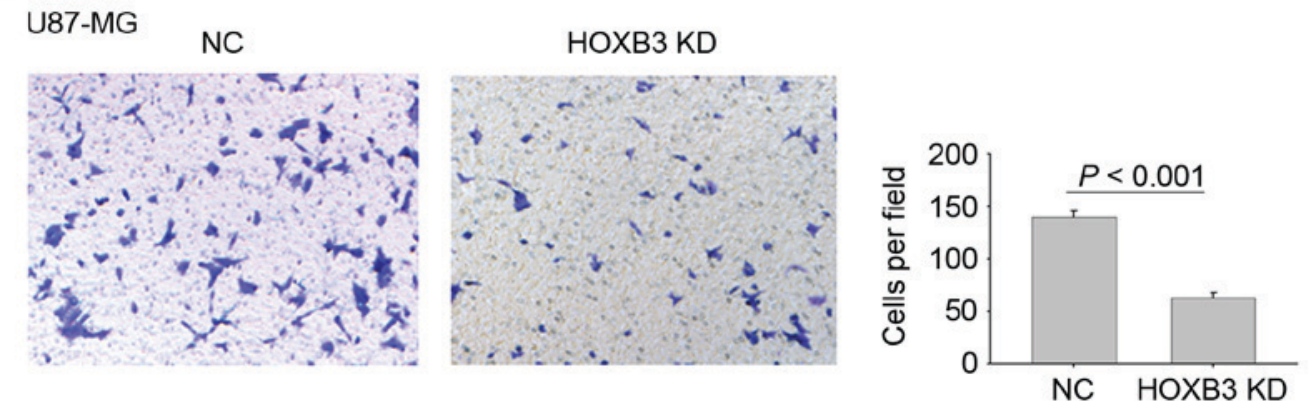

B
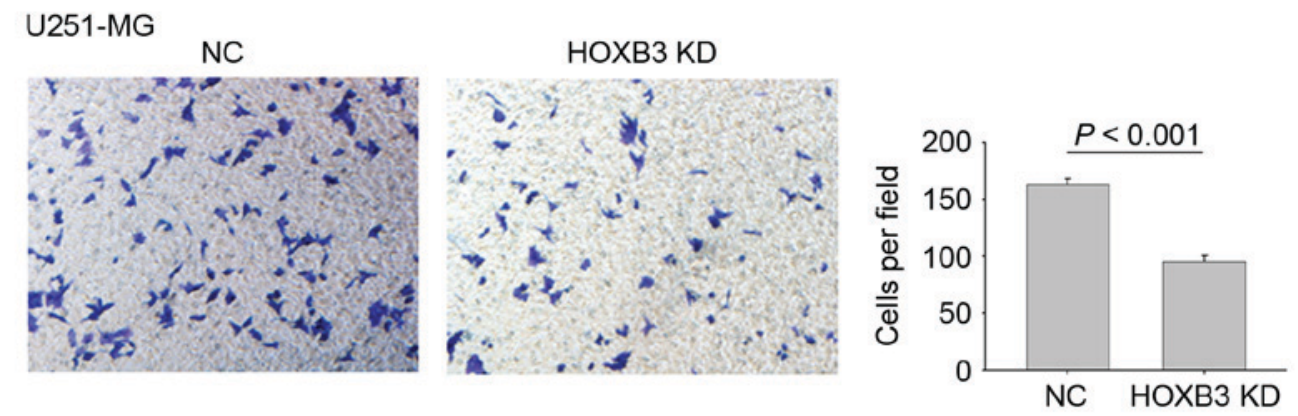

C

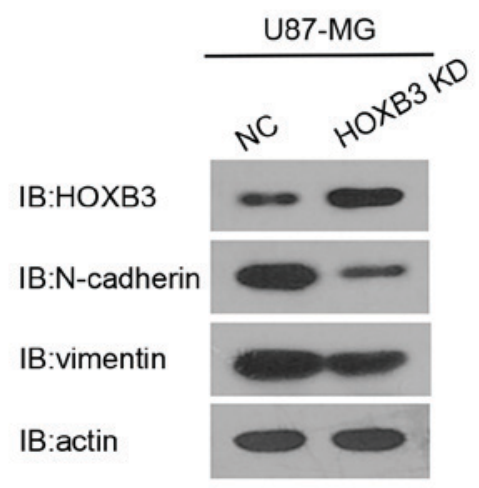

D

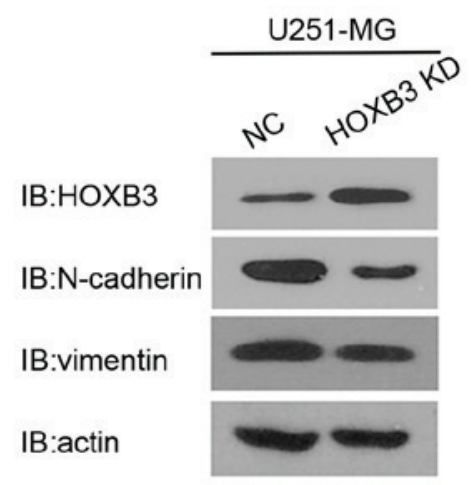

Figure 3. Silencing of HOXB3 inhibits GBM cell invasion. Cells were subjected to a Matrigel invasion assay. (A) HOXB3-KD U87-MG cells exhibited decreased invasiveness compared with their parental counterparts. (B) HOXB3-KD U251-MG cells exhibited decreased invasiveness compared with their parental counterparts. Expression levels of several epithelial and mesenchymal markers were analyzed in the HOXB3-KD cells and compared with their respective parental counterparts. (C) Western blot analysis of HOXB3 expression revealing upregulated protein level of E-cadherin and downregulated protein levels of N-cadherin and vimentin in HOXB3-KD U87-MG cells. (D) Western blot analysis of HOXB3 expression revealing upregulated protein level of E-cadherin and downregulated protein levels of N-cadherin and vimentin in HOXB3-KD U251-MG cells. GBM, glioblastoma; HOXB3-KD, homeobox B3-knockdown; NC, negative control.

result from triggering the increased expression of $\mathrm{N}$-cadherin and vimentin.

\section{Discussion}

High expression of HOXB3 has been reported in a variety of cancer types, including lung and colon cancer, and its relevance to poor prognosis of cancer has also been established $(13,15,17)$. However, the role for HOXB3 in GBM remains unknown. To the best of our knowledge, this is the first report to reveal that HOXB3 promotes cell proliferation and invasion in GBM.

GBM cells often invade into adjacent $1-2 \mathrm{~cm}$ of the normal brain tissue, which allows tumor cells to evade standard treatment interventions (2). Thus, there is a pressing requirement to define the molecular mechanisms responsible for invasion. The present study focused on HOXB3, and demonstrated that silencing of HOXB3 was able to inhibit tumor cell invasion in glioblastoma. These findings indicate that HOXB3 serves a role in GBM cell invasion. In this regard, it is unexpected in that HOXB3 has been reported to suppress or has no effect on cell invasion and migration. Weiss et al (25) observed that the specific silencing of HOXB3 was sufficient to reduce the migration of pancreatic cancer cells in vitro, and invasion and metastasis of pancreatic cancer in vivo, indicating that HOXB3 functions as a suppressor of cell invasion. In colorectal cancer, high expression of HOXB3 had no effect on cell invasion, but instead participates in regulation of cell proliferation (18). Therefore, the present findings increase the body of understanding regarding the role of HOXB3 in cancer. 
EMT is an important regulatory program by which transformed epithelial cells acquire enhanced invasiveness (26). Carcinoma cells that arise from normal epithelial cells acquire multiple attributes of EMT, including alterations to their shape, and abrogation of cell-cell interactions (14) (such as altered expression of cadherins), that enable their invasion. Whereas GBMs are non-epithelial tumors, highly invasive GBM cells necessarily undergo a switch to a more mesenchymal and aggressive state, referred to as an EMT-like program, similar to the classical EMT characterized in epithelial cells (27). The present study initially observed that silencing of HOXB3 led to a resulting change in cell shape, a typical sign of EMT, and concomitant reduction in invasiveness. Therefore, we hypothesized that reduced cell invasion may result from molecular events associated with the EMT-like program. The results of the present study indicated that expression of molecules associated with EMT, including N-cadherin and vimentin, were downregulated in the cancer cells that silence HOXB3 expression. By contrast, the epithelial marker E-cadherin was upregulated in the HOXB3-silencing cells. These findings are consistent with those obtained from previous studies $(28,29)$, in which GBM cell invasion is associated with the altered expression of key EMT-associated genes.

Although multiple examples of aberrantly high HOXB3 expression, combined with the data presented in the present report, demonstrate that HOXB3 has been implicated in tumor development and progression, its effect on cancerous phenotypes, particularly cancer cell proliferation, remains poorly characterized. Several findings otherwise provide evidence against the positive role of HOXB3 in cancer cell proliferation, as exemplified by: i) 19 malignant mammary cell lines, including MDA-MB-231 and MCF-7 cells, exhibited downregulated level of HOXB3 compared with the non-malignant SVCT cell line and human breast tissue; ii) malignant melanoma cell lines also exhibited downregulation of HOXB3 expression (30). Additionally, the biological effects of Hox genes vary and exhibit tissue specificity (31). It is therefore possible that the HOXB3 functions as a pro-proliferative factor depending on the cell type. In certain types of cancer, including leukemia, a set of Hox genes acting cooperatively promote clonal expansion (32). By contrast, Zhang et al (33) observed that forced expression of a single Hox gene can affect tumorigenesis in neuroblastoma. Coinciding with the latter, the results of the proliferation assay in the present study revealed that HOXB3 had a noticeable effect on cell growth. To further investigate the effect of HOXB3 signaling on cell proliferation, future studies should specifically examine which of the cell cycle genes may be regulated by the action of HOXB3.

In conclusion, the findings presented in the present study reveal that HOXB3 mediates cell proliferation and invasion in U87-MG and U251-MG cells, providing an insight into the active role of HOXB3 in GBM. The current study raises the possibility that HOXB3 acts as a potential therapeutic target, information that potentially contributes to GBM therapy in the future.

\section{Acknowledgements}

The authors are grateful to the National Natural Science Foundation of China (grant no. 81660502) for funding this study.

\section{References}

1. Bonavia R, Inda MM, Cavenee WK and Furnari FB: Heterogeneity maintenance in glioblastoma: A social network. Cancer Res 71: 4055-4060, 2011.

2. Hou LC, Veeravagu A, Hsu AR and Tse VC: Recurrent glioblastoma multiforme: A review of natural history and management options. Neurosurgical Focus 20: E5, 2006.

3. Gray S, Pandha HS, Michael A, Middleton G and Morgan R: HOX genes in pancreatic development and cancer. JOP 12: 216-219, 2011.

4. Scott MP: A rational nomenclature for vertebrate homeobox (HOX) genes. Nucleic Acids Res 21: 1687-1688, 1993.

5. Scott MP: Vertebrate homeobox gene nomenclature. Cell 71: 551-553, 1992.

6. Levine $\mathrm{M}$ and Hoey $\mathrm{T}$ : Homeobox proteins as sequence-specific transcription factors. Cell 55: 537-540, 1988.

7. Calvo R, West J, Franklin W, Erickson P, Bemis L, Li E, Helfrich B, Bunn P, Roche J, Brambilla E, et al: Altered HOX and WNT7A expression in human lung cancer. Proc Natl Acad Sci USA 97: 12776-12781, 2000.

8. Hung YC, Ueda M, Terai Y, Kumagai K, Ueki K, Kanda K, Yamaguchi H, Akise D and Ueki M: Homeobox gene expression and mutation in cervical carcinoma cells. Cancer Sci 94: 437, 2003.

9. Cantile M, Pettinato G, Procino A, Feliciello I, Cindolo L and Cillo $\mathrm{C}$ : In vivo expression of the whole HOX gene network in human breast cancer. Eur J Cancer 39: 257-264, 2003.

10. Vider BZ, Zimber A, Chastre E, Gespach C, Halperin M, Mashiah P, Yaniv A and Gazit A: Deregulated expression of homeobox-containing genes, HOXB6, B8, C8, C9, and $\mathrm{Cdx}-1$, in human colon cancer cell lines. Biochem Biophys Res Commun 272: 513-518, 2000.

11. Friedmann Y, Daniel CA, Strickland P and Daniel CW: Hox genes in normal and neoplastic mouse mammary gland. Cancer Res 54: 5981-5985, 1994.

12. Chen J, Zhu S, Jiang N, Shang Z, Quan C and Niu Y: HoxB3 promotes prostate cancer cell progression by transactivating CDCA3. Cancer Lett 330: 217-224, 2013.

13. Bodey B, Bodey B Jr, Gröger AM, Siegel SE and Kaiser HE: Immunocytochemical detection of homeobox B3, B4, and C6 gene product expression in lung carcinomas. Anticancer Res 20: 2711-2716, 2000.

14. Blaschuk OW and Devemy E: Cadherins as novel targets for anti-cancer therapy. Eur J Pharmacol 625: 195-198, 2009.

15. Palakurthy RK, Wajapeyee N, Santra MK, Gazin C, Lin L, Gobeil S and Green MR: Epigenetic silencing of the RASSF1A tumor suppressor gene through HOXB3-mediated induction of DNMT3B expression. Mol Cell 36: 219-230, 2009.

16. Chen H, Fan Y, Xu W, Chen J, Xu C, Wei X, Fang D and Feng Y: miR-10b inhibits apoptosis and promotes proliferation and invasion of endometrial cancer cells via targeting HOXB3. Cancer Biother Radiopharm 31: 225-231, 2016.

17. Almeida MI, Nicoloso MS, Zeng L, Ivan C, Spizzo R, Gafà R Xiao L, Zhang X, Vannini I, Fanini F, et al: Strand-specific miR-28-5p and miR-28-3p have distinct effects in colorectal cancer cells. Gastroenterology 142: 886-896.e9, 2012.

18. Mikheeva SA, Mikheev AM, Petit A, Beyer R, Oxford RG Khorasani L, Maxwell JP, Glackin CA, Wakimoto H, González-Herrero I, et al: TWIST1 promotes invasion through mesenchymal change in human glioblastoma. Mol Cancer 9: 194, 2010.

19. Cheng WY, Kandel JJ, Yamashiro DJ, Canoll P and Anastassiou D: A multi-cancer mesenchymal transition gene expression signature is associated with prolonged time to recurrence in glioblastoma. PLoS One 7: e34705, 2012.

20. Kotelevets L, Van-Hengel J, Bruyneel E, Mareel M, Van-Roy F and Chastre E: The lipid phosphatase activity of PTEN is critical for stabilizing intercellular junctions and reverting invasiveness. J Cell Biol 155: 1129-1135, 2001.

21. Xia M, Hu M, Wang J, Xu Y, Chen X, Ma Y and Su L: Identification of the role of Smad interacting protein 1 (SIP1) in glioma. J Neurooncol 97: 225-232, 2010.

22. Asano K, Duntsch CD, Zhou Q, Weimar JD, Bordelon D, Robertson $\mathrm{JH}$ and Pourmotabbed T: Correlation of N-cadherin expression in high grade gliomas with tissue invasion. J Neurooncol 70: 3-15, 2004.

23. Motta FJ, Valera ET, Lucio-Eterovic AK, Queiroz RG, Neder L, Scrideli CA, Machado HR, Carlotti-Junior CG, Marie SK and Tone LG: Differential expression of E-cadherin gene in human neuroepithelial tumors. Genet Mol Res 7: 295-304, 2008. 
24. Lu KV, Chang JP, Parachoniak CA, Pandika MM, Aghi MK, Meyronet D, Isachenko N, Fouse SD, Phillips JJ, Cheresh DA, et al: VEGF inhibits tumor cell invasion and mesenchymal transition through a MET/VEGFR2 complex. Cancer Cell 22: 21-35, 2012.

25. Weiss FU, Marques IJ, Woltering JM, Vlecken DH, Aghdassi A, Partecke LI, Heidecke CD, Lerch MM and Bagowski CP: Retinoic acid receptor antagonists inhibit mir-10a expression and block metastatic behavior of pancreatic cancer. Gastroenterology 137: 2136-2145.e1-7, 2009.

26. Polyak K and Weinberg RA: Transitions between epithelial and mesenchymal states: Acquisition of malignant and stem cell traits. Nat Rev Cancer 9: 265-273, 2009.

27. Hanahan D and Weinberg R: Hallmarks of cancer: The next generation. Cell 144: 646-674, 2011.

28. Kahlert UD, Nikkhah G and Maciaczyk J: Epithelial-to-mesenchymal(-like) transition as a relevant molecular event in malignant gliomas. Cancer Lett 331: 131-138, 2013.

29. Kahlert UD, Maciaczyk D, Doostkam S, Orr BA, Simons B Bogiel T, Reithmeier T, Prinz M, Schubert J, Niedermann G, et al: Activation of canonical WNT/ $\beta$-catenin signaling enhances in vitro motility of glioblastoma cells by activation of ZEB1 and other activators of epithelial-to-mesenchymal transition. Cancer Lett 325: 42-53, 2012
30. Svingen T and Tonissen KF: Altered HOX gene expression in human skin and breast cancer cells. Cancer Biol Ther 2: 518-523, 2003.

31. Shah N and Sukumar S: The Hox genes and their roles in oncogenesis. Nat Rev Cancer 10: 361-371, 2010.

32. Calvo KR, Sykes DB, Pasillas MP and Kamps MP: Nup98-HoxA9 immortalizes myeloid progenitors, enforces expression of Hoxa9, Hoxa7 and Meis1, and alters cytokine-specific responses in a manner similar to that induced by retroviral co-expression of Hoxa9 and Meis1. Oncogene 21: 4247-4256, 2002.

33. Zhang X, Hamada J, Nishimoto A, Takahashi Y, Murai T, Tada M and Moriuchi T: HOXC6 and HOXC11 increase transcription of S100beta gene in BrdU-induced in vitro differentiation of GOTO neuroblastoma cells into Schwannian cells. J Cell Mol Med 11: 299-306, 2007

This work is licensed under a Creative Commons Attribution-NonCommercial-NoDerivatives 4.0 International (CC BY-NC-ND 4.0) License. 